\title{
VORSCHAU 04
}

\section{ENTWICKLUNG}

\section{Skalierbare IT-Systeme bei \\ Car-to-X-Techniken}

Robustness Validation - Qualifizierungsmaßnahmen für funktionale Sicherheit

Most-Bus - Roadmap auf dem Prüfstand
Elektromotoren mit integrierter Leistungselektronik

Neues leitfähiges Polymer für Folien, Kondensatoren und Touchscreens

Virtualisierung von Steuergerätesoftware

\section{FORSCHUNG}

Softwaregesteuerte, radindividuelle Drehmomentenverteilung in einem batterieelektrischen E-Fahrzeug

48-V-Bordnetz - Status auf

Komponentenebene

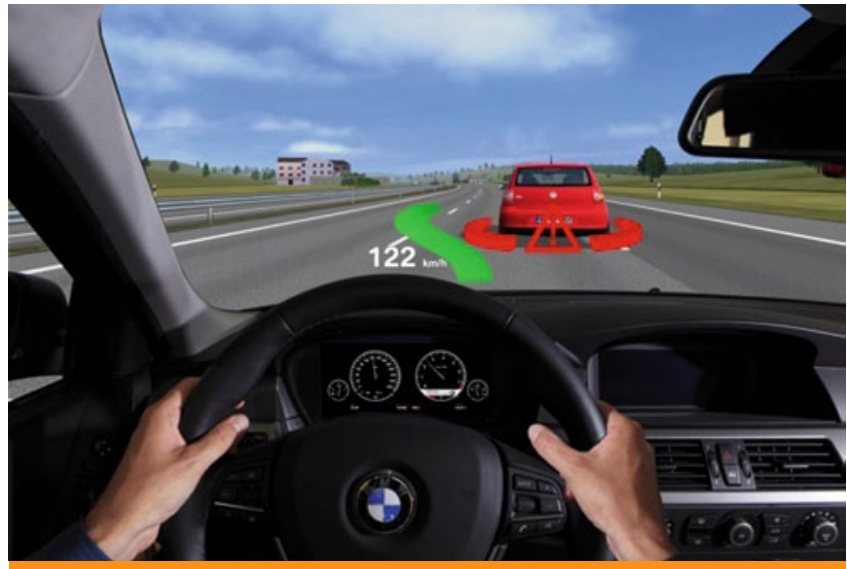

TITELTHEMA

OPTISCHE SYSTEME FÜR FAHRERASSISTENZ-FUNKTIONEN

Head-up-Displays (HUD) sind seit 2003 im Serieneinsatz. Geschwindigkeit, Warnungen und Navigationshinweise werden direkt ins Blickfeld des Fahrers projiziert und scheinen über der Motorhaube zu schweben. 2014 folgt die zweite HUD-Generation, erweitert mit der Projektion Augmented Reality (AR). Wenn beispielsweise vor einer drohenden Kollision gewarnt werden soll, kann jetzt das Hindernis entsprechend hervorgehoben und sogar eine Ausweichroute angezeigt werden, wenn die Sensorik des Fahrzeugs die Möglichkeit eines sicheren Spurwechsels erkannt hat.

Continental, einer der Zulieferer der ersten und der neuen Generation von Head-up-Displays, erklärt das Fahrerassistenzsystem und die Auslegung der Betriebsstrategien.

Die Firma RTT ist, was die HUD-Technik betrifft, ein Quereinsteiger. Der Anbieter von High-End-Software und Kreativleistungen für professionelle 3D-Visualisierung beschäftigte sich mit AR bisher in der Präsentationstechnik im Entwicklungsprozess von Fahrzeugen. Jetzt ist das Software-Know-how der Münchner bei der AR-HUD-Technik gefragt.

Im Interview mit Professor Dr.-Ing. Markus Maurer vom Institut für Regelungstechnik der Technischen Universität Braunschweig spricht ATZelektronik über die Einordnung der optischen Fahrerassistenzsysteme im Gesamtkontext Fahrersicherheit. 